\title{
The effect of levosimendan combined with 20\% lipid emulsion treatment on survival from bupivacaine induced toxicity in experiment
}

\author{
Gokahmetoglu $\mathrm{G}^{1}$, Aksu R², Bicer $\mathrm{C}^{2}$, Darcin $\mathrm{K}^{3}$, Ugur $\mathrm{F}^{2}$ \\ Department of Anesthesiology, Egitim Araştirma Hastanesi, Kayseri, Turkey.gunhangok@gmail.com
}

\begin{abstract}
Background: Recent reports demostrated that levosimendan improved post-resuscitation myocardial function in rat and pig models.

Materials and methods: Rabbits were randomized into 4 groups as 12 rabbits in each group. Bupivacaine 10 $\mathrm{mg} / \mathrm{kg}$ was injected as an intravenous bolus to all groups. Basic life support was performed by mechanical ventilation and manual external chest compressions. After $1 \mathrm{~min}$, animals in the group 1 received $1.5 \mathrm{ml} / \mathrm{kg}$ saline $0.9 \%$ solution, and animals in the groups 2 and 4 received $5 \mathrm{ml} / \mathrm{kg} 20 \%$ lipid emulsion for 1 min through the ear vein followed by continuous infusion at $0.25 \mathrm{ml} / \mathrm{kg} / \mathrm{min}$. Three additional boluses of $1.5 \mathrm{ml} / \mathrm{kg}$ lipid emulsion were repeated at 5 -min intervals. The group 3 received fluid resuscitation plus levosimendan $(3 \mu \mathrm{g} / \mathrm{kg} / \mathrm{min}) 1$ min after asystole and the group 4 received both levosimendan and lipid emulsion treatment. Return of spontaneous circulation and hemodynamic metrics were obtained in 20 minutes.

Results: The number of rabbits that survived after cardiac resuscitation was lower in the Groups $1(0 \%)$ and $3(33.3 \%)$ than in the Group $4(91.7 \%)$ with a statistically significant difference $(p<0.001)$. The number of rabbits that survived resuscitation was higher in the Group 4 than in the Group 2 (66.7 \%), though not with a statistically significant difference $(p=0.317)$. The median duration of cardiac arrest in the Group 4 was significantly shorter than that in the other three groups $(p<0.001)$.

Conclusions: In this rabbit model of bupivacaine-induced cardiac arrest, resuscitation with combined iv lipid emulsion and levosimendan was more efficacious than lipid alone (Tab. 3, Ref. 24). Text in PDF www.elis.sk. Key words: local anesthetic systemic toxicity, cardiovascular system, central nervous system, regional anesthesia, levosimendan, post-resuscitation, myocardial function, rat, pig, lipids, cardiopulmonary resuscitation, initial resuscitation success, bupivacaine.
\end{abstract}

Local anesthetic systemic toxicity (LAST), including complications of the cardiovascular and central nervous system (CNS), is a rare but devastating complication of regional anesthesia (1).

Although the incidence of LAST is less than $0.2 \%$ (2), LAST is difficult to treat and is potentially fatal. Lipid emulsion therapy is gaining acceptance as a part of the treatment of systemic local anesthetic toxicity (3). Intravenous lipid emulsion is now an accepted treatment for bupivacaine-induced cardiotoxicity with guidelines for lipid application endorsed by anesthetic and toxicologic societies internationally $(4,5)$.

Levosimendan is a novel inodilator agent that enhances myocardial performance without causing substantial changes in oxygen consumption. The combined positive inotropic and vasodilation effects of levosimendan relate to its effect on $\mathrm{Ca}^{2+}$ sensitiation and opening of $\mathrm{K}^{+}$channels (6). Recent reports demonstrated that le-

${ }^{1}$ Department of Anesthesiology, Egitim Araştirma Hastanesi, Kayseri, Turkey, ${ }^{2}$ Department of Anesthesiology, Erciyes University Medical Faculty, Kayseri, Turkey, and ${ }^{3}$ Department of Anesthesiology, Develi Devlet Hastanesi, Kayseri, Turkey

Address for correspondence: G. Gokahmetoglu, Research and Education Hospital Department of Anesthesiology , Kayseri, Turkey.

Phone: $+90.352 .3368884 / 1442$ vosimendan improved post-resuscitation myocardial function in swine and pig models $(7,8)$.

Our aim was to investigate whether the administration of levosimendan with lipid during cardiopulmonary resuscitation would improve the initial resuscitation success.

\section{Materials and methods}

Following approval from the Erciyes University Ethics Committee for Animal Experiments, 48 healthy male and female New Zealand rabbits were randomized into the 4 groups. Group $1(\mathrm{n}=$ 12): Bupivacaine $+0.9 \% \mathrm{NaCl}$, Group $2(\mathrm{n}=12)$ : Bupivacaine + lipid emulsion, Group $3(\mathrm{n}=12)$ : Bupivacaine + levosimendan, and Group $4(n=12)$ : Bupivacaine + lipid emulsion + levosimendan. Anesthesia was induced with $50 \mathrm{mg} / \mathrm{kg}$ ketamine (Ketalar, Mayne Pharma Ltd., New Zealand) and $4 \mathrm{mg} / \mathrm{kg}$ xylazin (Rompun, Bayer HealthCare, Germany) via i.m. injection. A three lead electrocardiogram (ECG) was cited for continuous monitoring. Animals were placed on a surgical board and venous cannulation of the both marginal ear veins were performed.-

Rabbits were ventilated with $100 \% \mathrm{O}_{2}$ for 3 minutes via face mask. Tracheal intubation was performed with a uncuffed 
endotracheal tube (ET) (Mallinckrodt Medical Inc., St. Louis, USA, size $2.5 \pm 0.5$ ), and pressure was controlled by mechanical ventilation (titrated to $15 \mathrm{~cm} \mathrm{H}_{2} \mathrm{O}$ inspiratory pressure) and instituted with $100 \% \mathrm{O}_{2}$ at 17 breaths/min via Nuffield series 200 pediatric ventilator (Penlon Ltd., Abington, England). Blunt dissection was employed to expose the left common carotid artery. The baseline and 20 minute arterial blood samples were taken for blood gases analysis. A saline-filled 20G cannula was advanced from the internal carotid to the proximal aorta and connected to a Hewlett Packard 78834 with a pressure transducer (Edwards Lifesciences, Irvine, CA, USA). A neonatal monitor (Hewlett Packard, Palo Alto, CA) was used for continuous arterial blood pressure monitoring.

\section{Bupivacaine arrest protocol}

Bupivacaine (AstraZeneca Ltd., New Zealand) $10 \mathrm{mg} / \mathrm{kg}$ (9, 10) was injected as an intravenous bolus for $5 \mathrm{~s}$ via the venous cannula in the marginal ear vein.

\section{Resuscitation protocol}

Following the induction of asystole, a 30-s non-intervention period designed to mimic clinical response time, was afforded. Basic life support (BLS) was performed by mechanical ventilation and manual external chest compressions (approximately $30 \%$ anterior - posterior chest diameter at 180 compressions per minute, timed by metronome) by an investigator blinded to all groups and arterial pressure information.

The time of asystole was defined as 0 time.

Animals in the saline group (Group 1) received $1.5 \mathrm{ml} / \mathrm{kg}$ saline $0.9 \%$ solution for $1 \mathrm{~min}$, followed by continuous infusion at 0.25 $\mathrm{ml} / \mathrm{kg} / \mathrm{min}$ via the ear vein. At 1 minute, animals from the Groups 2 and 4 received $1.5 \mathrm{ml} / \mathrm{kg} 20 \%$ lipid emulsion (Intralipid, Fresenius Kabi AB, Stockholm, Sweden) for $1 \mathrm{~min}$ via the ear vein, followed by continuous infusion at $0.25 \mathrm{ml} / \mathrm{kg} / \mathrm{min}$ in accordance with the recommendations of the Association of Anaesthetists of Great Britain and Ireland (AAGBI) and American Society of Regional Anesthesia (ASRA). In the absence of ROSC, or following repeated cardiac arrest in animals that displayed initial ROSC, 2 additional boluses of $1.5 / \mathrm{ml} / \mathrm{kg}$ intralipid were repeated at $5-\mathrm{min}$ intervals (equating to 6 and $11 \mathrm{~min}$, respectively). If, after another $5 \mathrm{~min}$ (time $16 \mathrm{~min}$ ), adequate circulation had not been restored, 1 additional bolus of $1.5 / \mathrm{ml} / \mathrm{kg}$ intralipid were repeated and the rate of infusion was increased to $0.5 \mathrm{ml} / \mathrm{kg} / \mathrm{min}$.
Animals in the Groups 3 and 4 received levosimendan $(3 \mu \mathrm{g} /$ $\mathrm{kg} / \mathrm{min}) 1$ minute after asystole via another ear vein.

With asystole, all animals received adrenaline at high dose $(100 \mu \mathrm{g} / \mathrm{kg})(10)$ every 5 minutes via the ear vein.

The return of spontaneous circulation (ROSC) was defined by the mean arterial pressure (MAP) higher or equal to $50 \mathrm{mmHg}$, with a restoration of the native heart rhythm for $120 \mathrm{~s}$ or longer. Resuscitative efforts were continued until 20 min (representing completion of the initial AAGBI infusion period), at which time all surviving animals were euthanized with an intravenous bolus injection of $300 \mathrm{mg}$ pentobarbital.

Necropsy was performed to confirm the correct placement of vascular catheters and the tracheal tube.

The primary outcome of this study was to assess the effect of levosimendan on 20 minutes survival after cardiac arrest due to iv bupivacaine.

\section{Statistical analysis}

The data were analyzed with the IBM SPSS Statistics v20 package (IBM Corporation, Somers, NY, USA). Shapiro-Wilk test was used to assess data distribeted normally or not. Normally distributed groups were evaluated by one-way analysis of variance. When the analysis of variance demonstrated a difference, the Student-Newman-Keuls method was employed to determine, which groups showed a difference. Abnormally distributed data were evaluated by the Kruskal-Wallis analysis. When the Kruskal-Wallis analysis demonstrated a difference, Dunn's multiple comparison test was used to identify, which groups were showing a difference. Categorical variables were compared with the Chi-square test. A $p$ value of $<0.05$ was recognized as statistically significant.

Power analysis was based on previous work in a similar model (9) with a predicted $50 \%$ rate of return of spontaneous circulation. To achieve a $90 \%($ alpha $=0.05$, beta $=0.10)$ instead of $50 \%$ survival rate by application of lipid emulsion for $20 \mathrm{~min}$, at least 12 rabbits were required in each group (version 10.1.6; ${ }^{\mathrm{a}}$ 1993-2009, MedCalc Software, Mariakerke, Belgium).

\section{Results}

Baseline animal characteristics and hemodynamic parameters are detailed in the Table 1. No statistically significant differences in heart rate, $\mathrm{MAP}, \mathrm{SpO}_{2}$ or rabbit weight were observed between the groups at the beginning of the experiment and at 20 minutes

Tab. 1. Animal characteristics and baseline haemodynamic variables.

\begin{tabular}{|c|c|c|c|c|c|}
\hline & $\begin{array}{c}\text { Group 1 } \\
(\mathrm{n}=12) \\
\text { Mean (SD) }\end{array}$ & $\begin{array}{c}\text { Group } 2 \\
(\mathrm{n}=12) \\
\text { Mean (SD) }\end{array}$ & $\begin{array}{c}\text { Group } 3 \\
(\mathrm{n}=12) \\
\text { Mean (SD) }\end{array}$ & $\begin{array}{c}\text { Group } 4 \\
(\mathrm{n}=12) \\
\text { Mean (SD) }\end{array}$ & $\mathrm{p}$ \\
\hline Weight (g) & $3805 \pm 314$ & $3675 \pm 743$ & $3666 \pm 374$ & $3525 \pm 850$ & 0.744 \\
\hline MAP (mmHg) (Basale) & $70 \pm 10$ & $64 \pm 13$ & $67 \pm 12$ & $70 \pm 10$ & 0.469 \\
\hline $\operatorname{MAP}(\mathrm{mmHg})(20 . \mathrm{min})$ & - & $75 \pm 46$ & $81 \pm 30$ & $87 \pm 32$ & 0.820 \\
\hline HR (beat/min) (Basale) & $176 \pm 6$ & $182 \pm 7$ & $180 \pm 6$ & $179 \pm 7$ & 0.177 \\
\hline HR (beat/min) (20.min) & - & $194 \pm 11$ & $196 \pm 14$ & $188 \pm 9$ & 0362 \\
\hline $\mathrm{SpO}_{2}(\%)(20 . \mathrm{min})$ & - & $87 \pm 9$ & $83 \pm 12$ & $92 \pm 10$ & 0.317 \\
\hline
\end{tabular}

Group1 = Control group; Group 2 = Lipid group; Group 3 = Levosimendan group; Group $4=$ Lipid + Levosimendan group, $p<0.05$ statistically significant 
Tab. 2. Arterial blood gas variables at baseline and 20 minutes.

\begin{tabular}{|c|c|c|c|c|c|c|c|c|c|c|}
\hline & \multicolumn{2}{|c|}{ Group $1(\mathrm{n}=12)$} & \multicolumn{2}{|c|}{ Group $2(n=12)$} & \multicolumn{2}{|c|}{ Group $3(n=12)$} & \multicolumn{2}{|c|}{ Group $4(n=12)$} & \multicolumn{2}{|c|}{$\mathrm{P}$} \\
\hline & Baseline & 20th $\min$ & Baseline & 20th min & Baseline & 20th $\min$ & Baseline & 20th min & Baseline & 20th min \\
\hline $\mathrm{pH}$ & $7.38 \pm 0.04$ & & $7.32 \pm 0.05$ & $7.05 \pm 0.12$ & $7.39 \pm 0.05$ & $7.12 \pm 0.21$ & $7.35 \pm 0.10$ & $7.08 \pm 0.05$ & 0.097 & 0.672 \\
\hline $\mathrm{PaO}_{2} \mathrm{mmHg}$ & $95 \pm 16$ & - & $78 \pm 20$ & $85 \pm 27$ & $85 \pm 21$ & $93 \pm 43$ & $82 \pm 14$ & $102 \pm 30$ & 0.128 & 0.545 \\
\hline $\mathrm{PaCO}_{2} \mathrm{mmHg}$ & $27 \pm 3$ & - & $32 \pm 1$ & $33 \pm 1$ & $29 \pm 4$ & $24 \pm 1$ & $33 \pm 1$ & $31 \pm 5$ & 0.359 & 0.384 \\
\hline $\mathrm{HCO}_{3}^{2}(\mathrm{mmol} / \mathrm{L})$ & $17 \pm 1$ & - & $15 \pm 5$ & $9 \pm 3$ & $20 \pm 5$ & $9 \pm 4$ & $17 \pm 4$ & $9 \pm 2$ & 0.086 & 0.171 \\
\hline Lactate $(\mathrm{mmol} / \mathrm{L})$ & $6 \pm 2$ & - & $6.9 \pm 3$ & $7.8 \pm 3$ & $5.3 \pm 2$ & $7.4 \pm 1$ & $6.5 \pm 2$ & $7.7 \pm 2$ & 0.424 & 0.163 \\
\hline Base excess & $-8.4 \pm 3$ & - & $-10.3 \pm 4$ & $-20 \pm 3$ & $-6.3 \pm 4$ & $-22 \pm 4$ & $-7.2 \pm 4$ & $-19.5 \pm 2$ & 0.075 & 0.342 \\
\hline
\end{tabular}

Group1 = Control group; Group $2=$ Lipid group; Group $3=$ Levosimendan group; Group $4=$ Lipid + Levosimendan group, $p<0.05$ statistically significant

Tab. 3. Return of spontaneous circulation, spontaneous breathing, return time, number of arrests and duration of arrest of all groups.

\begin{tabular}{|c|c|c|c|c|c|}
\hline & Group $1(\mathrm{n}=12)$ & Group $2(\mathrm{n}=12)$ & Group $3(\mathrm{n}=12)$ & Group $4(n=12)$ & $\mathrm{p}$ \\
\hline Return of spontaneous circulation (n) (\%) & $0(0)$ & $8(66.7)+$ & $4(33.3)$ & $11(91.7)+@$ & $<0.001$ \\
\hline Return time(minute) (median) $(25-75 \%)$ & - & $7(2-7.25)$ & $10(6.5-15)$ & $2(2-3.75) *$ & 0.023 \\
\hline Numbers of arrests while alive (median) (min-max) & - & $1(1-2)$ & $1(1-3)$ & $2(1-3)$ & 0.058 \\
\hline Duration of arrest (median) $(25-75 \%)$ & $20(20-20)$ & $7.5(3.5-20)^{* *}$ & $20(15-20)$ & $4(3-5) * *+$ & $<0.001$ \\
\hline
\end{tabular}

Group1 = Control group; Group 2 = Lipid group; Group $3=$ Levosimendan group; Group $4=$ Lipid + Levosimendan group, +significantly high compared to the control group, @ significantly high compared to the levosimendan group, * significantly low compared to the control group, ** significantly low compared to the control and levosimendan groups, $\ddagger$ significantly high compared to the lipid group

$(\mathrm{p}>0.05)$ (Tab. 1). Furthermore, no significant differences in $\mathrm{pH}$, $\mathrm{paO}_{2}, \mathrm{paCO}_{2}, \mathrm{HCO}_{3}$, lactate, or base excess of the rabbits were observed between the groups at the beginning of the experiment and at 20 minutes $(\mathrm{p}>0.05)$ (Tab. 2).

All animals in all groups experienced asystole following bupivacaine injection.

None of the rabbits in the Group 1 exhibited ROSC within the 20 -minute follow-up period. However, $8(66.7 \%)$ rabbits in the Group 2, 4 rabbits (33.3\%) in the Group 3 and 11 (91.7\%) rabbits in the Group 4 exhibited ROSC and were recognized as survivors at the end of 20 minutes $(p<0.001)$ (Tab. 3). The number of rabbits that survived cardiac resuscitation was lower in the Groups 1 and 3 than in the Group 4, with a statistically significant difference $(\mathrm{p}<$ 0.001 ) (Tab. 3). The number of rabbits that survived resuscitation was higher in the Group 4 than in the Group 2, although the difference was not statistically significant difference $(p=0.314)$ (Tab. 3$)$.

The median duration of cardiac arrest for the Group 4 was significantly shorter than in the other groups, while that of the Group 2 was also significantly lower than that of the Groups 1 and $3(\mathrm{p}<0.001)$ (Tab. 3).

The median time to initial ROSC was $7 \mathrm{~min}$ in the Group 2, 10 $\min$ in the Group 3, and 2 min in the Group $4(p=0.023)$. None of the rabbits in the Group 1 exhibited ROSC (Tab. 3). The median time to initial response for resuscitation was shorter in the Group 4 than in the other groups, and the difference between the Group 4 and the Group 3 was statistically significant $(\mathrm{p}<0.05)$ (Tab. 3 ).

There was no significant difference between the Groups 2, 3 and 4 with regard to the rate of cardiac arrest during the 20-minute resuscitation period $(\mathrm{p}=0.058)($ Tab. 3$)$.

\section{Discussion}

Local anesthetic systemic toxicity is a rare but potentially catastrophic complication of regional anesthesia. Reports from animal studies (11) and case reports in humans (12) suggest lipid emulsion as a potential antidote that could reduce cardiovascular mortality of local anesthetic intoxication. The Association of Anaesthesists of Great Britain and Ireland recommends that all departments administering potentially toxic doses of LA should have lipid emulsion immediately available. This treatment protocol gained wide acceptance (3), and many anesthesists keep lipid emulsion at hand when performing regional anesthesia and consider giving lipid emulsion as first-line treatment in the case of LA intoxication (13). However, the exact mechanism of action of lipid emulsions in the treatment of LAST is not completely understood.

This study compared the effectiveness of lipid emulsion, levosimendan, and levosimendan administered after lipid emulsion in rabbits that received bupivacaine. The application of levosimendan to reduce mortality of LA intoxication has been reported $(8,14-16)$. To date, there are no published data comparing the effectiveness of lipid + levosimendan.

In this study, all of the rabbits in the placebo group died. Four and 8 rabbits died in the Groups 2 and 3, respectively, and a single rabbit died in the Group 4. Although there was no significant difference between the groups, most of the rabbits in the levosimendantreated group survived after lipid emulsion.

Tsagalou et al (17) reported cardiac arrest in a young man with idiopathic dilated cardiomyopathy. After a prolonged resuscitation effort with epinephrine, norepinephrine, and dobutamine, the administration of levosimendan seemed to aid in ROSC. The vasopressor combination of vasopressin and epinephrine compared with lipid emulsion in a bupivacaine-induced cardiac arrest in a pig model resulted in higher coronary perfusion pressure during CPR and better survival rates (18).

In this study, 8 of the lipid-treated rabbits survived and 4 of the 8 rabbits regained spontaneous breathing. Eleven of the rabbits treated with levosimendan after lipid emulsion survived, and all 11 rabbits returned spontaneous breathing. There was a statistically significant difference between groups regarding the return of spontaneous breathing. A pronounced, peripheral vasodilation effect of levosimendan has been described in previous studies (19). Therefore, the administration of levosimendan may cause vaso- 
dilation and thus restore blood flow to the underperfused cerebral parenchyma (7). Witt et al (20) showed that levosimendan to have significant vasodilation activity in the pulmonary vascular bed of the cat and also suggested that levosimendan may be useful in the treatment of disorders with increased pulmonary pressure. In our study, rabbits treated with levosimendan after lipid emulsion returned to spontaneous breathing.

Previous studies in an animal model of cardiac arrest demonstrated that coronary perfusion pressure (CPP) and ROSC were significantly improved by the coadministration of epinephrine + atenolol and epinephrine + levosimendan compared to epinephrine alone $(7,21)$. Huang et al $(22)$ found that levosimendan improved post-resuscitation myocardial dysfunction compared to dobutamine in a pig model of VF and closed-chest resuscitation. Xanthos et al (8) found that the administration of levosimendan and epinephrine was better than epinephrine alone regarding post-resuscitation myocardial function and that it significantly decreased the elevated serum levels of biomarkers for cerebral ischemia. In our study, the return time of spontaneous circulation was shorter in the rabbits treated with levosimendan after lipid emulsion than the lipid-treated rabbits or the levosimendantreated rabbits.

The bupivacaine-induced block of sodium channels develops primarily during the action potential plateau; therefore, levosimendan's shortening of the potential duration of cardiac action may enhance the speed of recovery of the sodium channels (23, 24). Toller et al (15) reported that the presence of levosimendan increased the potency of epinephrine and that the presence of epinephrine increased the potency of levosimendan, concerning myocardial contractility. Krummikly et al (16) reported that levosimendan stabilized the hemodynamics of a cardiac arrest patient treated with epinephrine. Such findings have led to the use of levosimendan, which is devoid of arrhythmogenic activity, to enhance myocardial contractility without increasing myocardial oxygen consumption and thereby increase the recovery of cardiac output in individuals experiencing cardiac arrest. In our study, the arrest duration of rabbits treated with levosimendan after lipid emulsion was shorter than lipid-treated rabbits, and this difference was statistically significant.

Therefore, we suggest that the coadministration of lipid and levosimendan benefits post-resuscitation myocardial function and reduces LAST. We conclude that the suggested therapeutic combination of epinephrine, lipid, and levosimendan to be more efficacious than the combinations of epinephrine and lipid, or epinephrine and levosimendan for post-resuscitation myocardial function and for the reduction of LAST.

A major limitation of this study was an evaluation time of only $20 \mathrm{~min}$. While a survival advantage of lipid application was documented within this duration, we are unable to comment on longer-term outcomes or eventual neurologically intact recovery.

Another limitation of this study was that we ignored the confounding effect of the ketamine and xylazine anesthetics and possible interactions between these drugs and lipid emulsion, levosimendan.

\section{Conclusion}

In conclusion, in this rabbit model of bupivacaine-induced cardiac arrest followed by resuscitation with combined iv, lipid emulsion and levosimendan was efficacious than lipid administration alone. Further and larger studies are required to compare lipid versus lipid + levosimendan treatment and to define the role of levosimendan in lipid-based resuscitation from cardiac arrest induced by local anesthetic.

\section{References}

1. Ozcan M, Weinberg G. Update on the use of Lipid Emulsions in Local Anesthetic Syste mic Toxicity: A Focus on Differential Efficacy and Lipid Emulsion as Part of Advanced Cardiac Life Support. Internat Anesth Clin 2011; 49 (4) : 91-103.

2. Auroy Y, Benhamou D, Bargues L et al. Major complications of regional anesthesia in France. The SOS regional anesthesia notline service. Anesthesiology 2002; 97: 1274-1280.

3. Picard J, Ward C, Zumpe R, Meek T, Barlow J, Griffiths H. Guidelines and The adoption of lipid rescue therapy for local anaesthetic toxicity. Anaesthesia 2009; 64: 122-125.

4. The Association of Anaesthetists of Great Britain and Ireland. http: www. aagbi.org/publications/guidelines.htm.(accessed 20/10/2009).

5. Washington Poisons Center. http://www.wpc.org/pdf/guideline/Intralipids.pdf.(accessed 04/03/2010).

6. Nieminen MS, Pollesello P, Vajda G, Papp Z. Effects of Levosimendan on the Energy Balance: Preclinical and Clinical Evidence. J Cardiovasc Pharmacol 2009; 53 (4): 302-310.

7. Koudouna E, Xanthos T, Bassiakou E et al.Levosimendan improves the initial outcome of cardiopulmonary resuscitation in a swine model of cardiac arrest. Acta Anaesth Scand 2007; 51: 1123-1129.

8. Xanthos T, Bassiakou E, Koudouna E et al. Combination pharmacotherapy in the treatment of experimental cardiac arrest. Amer J Emerg Med 2009; 27: 651-659.

9. Cave G, Harvey M, Prinde G, Lahner D, Desmet J. Effect of hypertonic saline on electrocardiography QRS of duration in rabbit model of bupivacaine toxicity resuscitated by intravenous lipid. Anaesthesia 2010; 65: 792-798.

10. Harvey M, Cave G, Prince G, Lahner D. Epinephrine injection in lipid-based resuscitation from bupivacaine-induced cardiac arrest: transient circulatory return in rabbits. Anesth Analg 2010; 111: 791-796.

11. Weinberg G, Ripper R, Feinstein DL et al. Lipid emulsion infusion rescues dogs from bupivacaine-induced cardiac toxicity. Reg Anesth Pain Med 2003; 28: 198-202.

12. Rosenblatt MA, Abel M, Fischer GW, Itzkovich CJ, Eisenkraft JB. Successful use of a $20 \%$ lipid emulsion to resuscitate a patient after a presumed bupivacaine-related cardiac arrest. Anesthesiology 2006; 105: 217-218.

13. Spence AG. Lipid reversal of central nervous system symptoms of bupivacaine toxicity. Anesthesiology 2007; 107: 516-517.

14. Aittomäki J, Liuhanen S, Sallisalmi M, Salmenperä MT, Heavner JE, Rosenberg PH. The Effect of Levosimendan on Bupivacaine- induced Severe Myocardial Depression in Anesthetized Pigs. Reg Anesth Pain Med 2010; 35 (1): 34-40. 
15. Toller W, Wolkart G, Stranz C, Metzler H, Brunner F. Contractile action of levosimendan and epinephrine during acidosis. Eur J Pharmacol 2005; 507: 199-209.

16. Krumnikl JJ, Toller WG, Prenner G, Metzler H. Beneficial outcome after prostoglandin induced post-partum cardiac arrest using levosimendan and extracorporeal membrane oxygenation. Acta Anestesiol Scand 2006; 50: 768-770.

17. Tsagalou EP, Nanas JN. Resuscitation from epinephrine resistant electromechanical dissociation facilitated by levosimendan in a young man with idiopathic dilated cardiomyopathy. Resuscitation 2006; 68: 147-149.

18. Mayr VD, Mitterschiffthaler L, Neurauter A et al. A Comparison of the Combination of Epinephrine and Vasopressin with Lipid Emulsion in a Porcine Model of Asphyxial Cardiac Arrest After Intravenous Injection of Bupivacaine. Anesth Analg 2008;106: 1566-1571.

19. Tassani $P$, Schad $H$, Heimisch $W$ et al. Effect of the calcium sensitizer levosimendanon the performance of ischaemic myocardium in anaesthetised pigs. Cardiovasc Drugs Ther 2002; 16: 435-441.
20. De Witt BJ, Ibrahim IN, Bayer E et al. An Analysis of Responses to Levosimendan in the Pulmonary Vascular Bed of the Cat. Anesth Analg 2002; 94: 1427-1433.

21. Bassiakou E, Xanthos T, Koudouna E, et al. Atenolol in combination with epinephrine improves the initial outcome of cardiopulmonary resuscitation in a swine model of ventricular fibrillation. Am J Emerg Med 2008; 26: 578-584.

22. Huang L, Weil MH, Tang W, Sun S, Wang J. Comparison between dobutamine and levosimendan for management of postresuscitation myocardial dysfunction. Crit Care Med 2005; 33: 487-491.

23. Stehr SN, Christ T, Rasche B et al. The effect of levosimendan on myocardial function in ropivacaine toxicity in isolated guinea pig heart preparations. Anesth Analg 2007; 105: 641-647.

24. Michaels AD, McKeown B, Kotsal M et al. Effects of intravenous levosimendan on human coronary vasomotor regulation, left ventricular wall stres, and myocardial oxygen uptake. Circulation 2005; 11: 1504-1509.

Received December 17, 2012. Accepted February 28, 2014. 